\title{
Spin-Orbit Interaction Induced by Heavy-Ion Projectile Excitation
}

\author{
H. Nishioka, R. C. Johnson, and J. A. Tostevin \\ Department of Physics, University of Surrey, Guildford, Surrey, England \\ and \\ K. -I. Kubo \\ Nuclear Physics Laboratory, University of Oxford, Oxford OX1 3RH, England, and Department of Physics, \\ Tokyo Metropolitan University, Setagaya-ku, Tokyo 158, Japan ${ }^{(a)}$ \\ (Received 30 November 1981)
}

\begin{abstract}
It is shown that, for heavy-ion projectiles with a cluster structure, effects due to projectile excitation generate a large effective spin-orbit interaction which can drastically alter the predictions for vector analyzing powers obtained from folding model spin-orbit potentials. Coupled-channels calculations of this effect for ${ }^{6} \mathrm{Li}$ and ${ }^{7} \mathrm{Li}$ scattering from ${ }^{58} \mathrm{Ni}$ at $E_{\text {c.m. }} \sim 20 \mathrm{MeV}$ give a good account of experimental data.

PACS numbers: $24.70 .+\mathrm{s}, 25.70 . \mathrm{Bc}, 25.70 . \mathrm{Hi}$
\end{abstract}

Recently there has been growing interest in the heavy -ion spin-orbit (SO) interaction. ${ }^{1-12}$ It has been found that SO potentials predicted by folding models ${ }^{1-7}$ are in many cases very different from the phenomenological SO potentials required by the data.

Measured vector analyzing powers, $i T_{11}$, for ${ }^{6} \mathrm{Li}$ elastic scattering from ${ }^{58} \mathrm{Ni}$ at $E_{\text {c. } \mathrm{m}_{0}}=21 \mathrm{MeV}$ (Ref. 3) and from ${ }^{4} \mathrm{He}$ at $E_{\mathrm{c} . \mathrm{m}_{*}}=6-9 \mathrm{MeV}$ (Ref. 8) have been found to have much larger magnitudes than predicted by folding models, ${ }^{2,3,5,7}$ although the folding models did give the gross features of the observed analyzing powers for a wide range of targets from ${ }^{12} \mathrm{C}$ to ${ }^{58} \mathrm{Ni}$. The phenomenologi cal SO potentials for ${ }^{13} \mathrm{C},{ }^{8}{ }^{15} \mathrm{~N},{ }^{10}$ and ${ }^{19} \mathrm{~F}$ projectiles, ${ }^{11}$ determined from transfer reactions and spin-flip probability measurements, were also much larger than those predicted. ${ }^{1-6}$ Moreover, the ${ }^{7} \mathrm{Li}+{ }^{58} \mathrm{Ni}$ vector analyzing power at $E_{\mathrm{c} . \mathrm{m}}$. $=18 \mathrm{MeV}$ was found $^{12}$ to have the opposite sign to that predicted by a folding model SO potential. ${ }^{5}$ Whereas the folding model SO potentials for ${ }^{6} \mathrm{Li}$ and ${ }^{7} \mathrm{Li}$ are of the same sign, ${ }^{5}$ the phenomenological SO potentials are of the opposite sign for ${ }^{6} \mathrm{Li}$ $+{ }^{58} \mathrm{Ni}$ and ${ }^{7} \mathrm{Ni}+{ }^{58} \mathrm{Ni}$ scattering.

In this Letter, strong coupling to a projectileexcited channel is shown to produce an effective projectile SO interaction with sign and magnitude similar to that required by experiment. The mechanism to be discussed is due to the exis tence of states with a well-defined cluster structure in the low-lying spectrum of the projectile and involves primarily the central parts of the individual cluster-target potentials. As such, the mechanism is a very general one and may have implications beyond the context mentioned in the last paragraph. The present discussion will be confined to the scattering of nuclei with spin from spinless targets at incident energies of a few megaelectronvolts per nucleon.

The scattering of ${ }^{6} \mathrm{Li}$ and ${ }^{7} \mathrm{Li}$ from an arbitrary spinless target is considered for definiteness. The ${ }^{6} \mathrm{Li}\left({ }^{7} \mathrm{Li}\right)$ nucleus is known ${ }^{13}$ to have a welldeveloped $d+\alpha(t+\alpha)$ cluster structure. The relative orbital angular momentum, $l$, between $d(t)$ and $\alpha$ is $0(1)$ in the ground state, $I^{\pi}=1^{+}\left(\frac{3}{2}^{-}\right)$, and $2(1)$ in the first excited state, $I^{\pi}=3^{+}, 2.18$ $\mathrm{MeV}\left(\frac{1}{2}^{-}, 0.48 \mathrm{MeV}\right)$. This projectile-excited inelastic channel is strongly coupled to the elastic channel through the quadrupole interaction between ${ }^{6} \mathrm{Li}\left({ }^{7} \mathrm{Li}\right)$ and the target. The effect of this coupling on the elastic scattering can be understood in the following classical discussion.

The case of ${ }^{6} \mathrm{Li}$ scattering is considered first. The spin-dependent part of the $d$-target interaction is much smaller than the central part and will be neglected. With this assumption the deuteron spin $\vec{S}$ is a constant of the motion and is equal to the ${ }^{6} \mathrm{Li}$ spin $\overrightarrow{\mathrm{I}}$ in the ground state. The $3^{+}$state of ${ }^{6} \mathrm{Li}$ has $l=2$ and therefore $\vec{I}$ is aligned with $\overrightarrow{\mathrm{S}}$. For an incident ${ }^{6} \mathrm{Li}$ with orbital angular momentum $\overrightarrow{\mathrm{L}}$ relative to the target, its total angular momentum is $\vec{J}=\overrightarrow{\mathrm{L}}+\overrightarrow{\mathrm{I}}$. In the classical limit conservation of $\vec{J}$ and $\vec{S}$ implies that the orbital angular momentum $\overrightarrow{\mathrm{L}}^{\prime}$ of the ${ }^{6} \mathrm{Li}$ intermediate excited state relative to the target must be $L^{\prime}=L$ -2 if $J=L+I$, and $L^{\prime}=L+2$ if $J=L-I$. For $J$ $=L, \overrightarrow{\mathrm{S}}$ and $\overrightarrow{\mathrm{I}}$ must be parallel to the reaction plane and $L^{\prime}=L$. There exists, therefore, a direct correspondence between $J$ and $L^{\prime}$ for purely kinematical reasons.

The intermediate channel of interest has $Q<0$, and hence, in grazing partial waves, channels with $L^{\prime}=L-2$ will be strongly favored over chan- 
nels with $L^{\prime}=L$ or $L+2$ because of centrifugal barrier effects. Together with the above kinematical argument this means that coupling to the inelastic channel is most effective if $J=L+I$ and least effective if $J=L-I$, i.e., the effect of the coupling on the elastic channel depends strongly on $J$ for a given $L$ and therefore is equivalent to a SO interaction with additional central and tensor terms.

In the case of ${ }^{7} \mathrm{Li}$ scattering, a similar classical discussion is possible, but now $\vec{I}$ and $\vec{S}$, the triton spin, are aligned in the ground state of ${ }^{7} \mathrm{Li}$ and antialigned in the excited state. Thus, the correspondence between $J$ and $L^{\prime}$ is opposite to that in the ${ }^{6} \mathrm{Li}$ case; namely $L^{\prime}=L+2$ if $J=L$ $+I, L^{\prime}=L-2$ if $J=L-I$, and $L^{\prime}=L$ for other $J$ values. As a result the effective $\mathrm{SO}$ interaction generated has opposite sign to that for ${ }^{6} \mathrm{Li}$ and the effect of projectile excitation on the elastic $i T_{11}$ for ${ }^{7} \mathrm{Li}$ scattering is expected to be opposite to that for ${ }^{6} \mathrm{Li}$, precisely as required by the experimental data.

Further insight into this effective SO interaction can be obtained in the adiabatic limit ${ }^{14}$ to the dynamic polarization potential induced by the excitation mechanism. For quadrupole excitation to intermediate states with $L^{\prime}=L-2$, this gives ${ }^{15}$

$$
\begin{aligned}
V_{\mathrm{SO}}{ }^{L}(R)= & \frac{3}{4} \frac{\left[6+I(I+1)-I^{\prime}\left(I^{\prime}+1\right)\right]}{I(I+1)(2 I+1)} \\
& \times \frac{(L-1)}{(2 L+1)(2 L-1)} \frac{\bar{V}_{I^{\prime} I}{ }^{(2)}(R)^{2}}{E_{\mathrm{exc}}},
\end{aligned}
$$

where $I^{\prime}$ and $E_{\text {exc }}$ are the spin and excitation energy of the projectile excited state, respectively, and $\bar{V}_{I^{\prime} I}{ }^{(2)}(R)$ is the channel coupling potential. The change in sign between ${ }^{6} \mathrm{Li}$ and ${ }^{7} \mathrm{Li}$ predicted by the semiclassical argument appears in the quantal discussion through the change of sign of the factor $6+I(I+1)-I^{\prime}\left(I^{\prime}+1\right)$ in Eq. (1).

Coupled-channels calculations for ${ }^{6} \mathrm{Li}$ and ${ }^{7} \mathrm{Li}$ scattering from ${ }^{58} \mathrm{Ni}$ at $E_{\text {c.m. }}=20.7$ and $18.1 \mathrm{MeV}$, respectively, have been performed, including the first projectile-excited-state channel, in order to investigate quantitatively this projectileexcitation effect on the elastic $i T_{11}$ and the validity of the qualitative discussion above.

All the diagonal and coupling potentials were obtained by the single folding procedure to avoid ambiguities arising from phenomenological parameters. The elementary $d-, t-$, and $\alpha-{ }^{58} \mathrm{Ni}$ interactions were taken from Refs. 16, 17, and 18 , respectively, and reproduce the respective elastic scattering data. The cluster ${ }^{58} \mathrm{Ni} \mathrm{Cou-}$ lomb potentials are also folded in. The relative wave functions of the $d(t)$ and $\alpha$ in ${ }^{6} \mathrm{Li}\left({ }^{7} \mathrm{Li}\right)$ were taken to be $2 S(2 P)$ and $1 D(2 P)$ states of a Woods-Saxon potential, ${ }^{19}$ for the ground and excited states, respectively. The ground-state wave functions obtained have similar shapes to those calculated microscopically, ${ }^{13}$ and the ${ }^{7} \mathrm{Li}$ wave function also reproduces the experimental $Q$ moment. As the excited state of ${ }^{6} \mathrm{Li}$ is a resonance state, the relative $d-\alpha$ wave function was smoothly cut off for cluster separations larger than $20 \mathrm{fm}$.

All terms of the interaction which arise in the folding procedure were included in the coupledchannels calculation, except for the coupling term which results from the $d-(t-)^{58} \mathrm{Ni} \mathrm{SO}$ potential. This term is considerably smaller than the coupling term due to the central potentials.

Calculated results for the elastic cross section and $i T_{11}$ in ${ }^{6} \mathrm{Li}+{ }^{58} \mathrm{Ni}$ scattering are shown as the solid curves in Fig. 1. For comparison, the results of a one-channel calculation are shown (dashed curves). As is clear from the figure, the folding-model SO potential produces only very small $i T_{11}$. The inelastic coupling effect produces a positive contribution to the $i T_{11}$, and gives results in good agreement with the data.

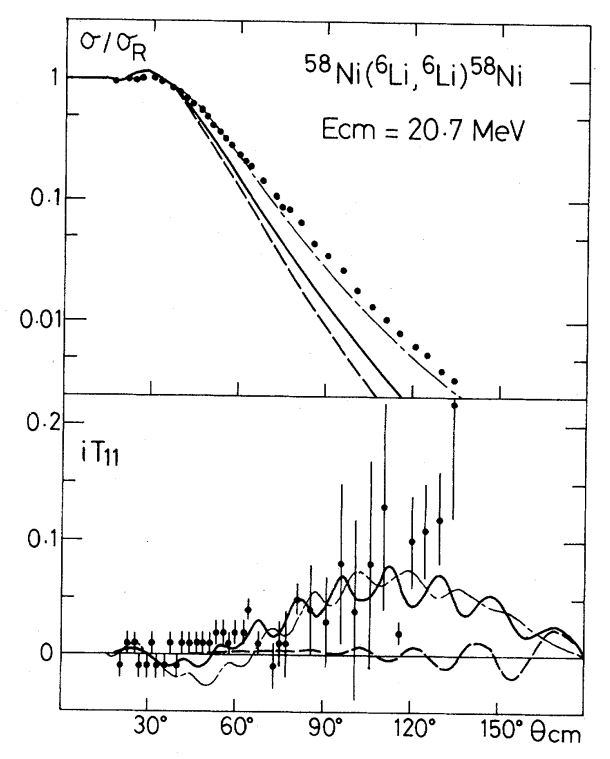

FIG. 1. Calculated results of the differential cross section (ratio to Rutherford) and vector analyzing power for ${ }^{6} \mathrm{Li}+{ }^{58} \mathrm{Ni}$ elastic scattering. The data are from Ref. 3. Dashed curves, solid curves, and dashdotted curves are the results of the one channel, coupled-channels, and renormalized coupled-channels calculations, respectively. 
According to the classical discussion, the spin dependence of the projectile-excitation mechanism arises because of the correspondence of the values of $J$ and $L^{\prime}$ with a fixed $L$ and also the dominance of the $L^{\prime}=L-2$ intermediate inelastic channels. This picture is confirmed for ${ }^{6} \mathrm{Li}$ and ${ }^{7} \mathrm{Li}$ scattering by the coupled-channels calculations. The calculated inelastic $T$-matrix elements have, in the grazing partial waves, larger magnitudes when $J, L^{\prime}$, and $L$ are in the above stated relation and also when $L^{\prime}=L-2$ than in other cases. In addition, the elastic $S$-matrix elements show the $J$ dependence expected from an effective SO potential of the form of Eq. (1).

In the ${ }^{7} \mathrm{Li}$ case, additional considerations arise because the folding-model potential in the groundstate channel has rank-2 $\left(T_{R}\right)$ and rank-3 tensor ter $\mathrm{ms}^{20}$ in addition to central and SO terms. Since the calculated $T_{R}$ potential is very large, its effect on $i T_{11}$ has been considered to be important. ${ }^{7}$ This effect is investigated here within a one-channel calculation. The dotted curves in Fig. 2 show the calculated elastic cross section and $i T_{11}$ for ${ }^{7} \mathrm{Li}+{ }^{58} \mathrm{Ni}$ scattering when only the central and SO terms of the potential are included. As pointed out in by Tungate et al. ${ }^{12}$ the calcu-

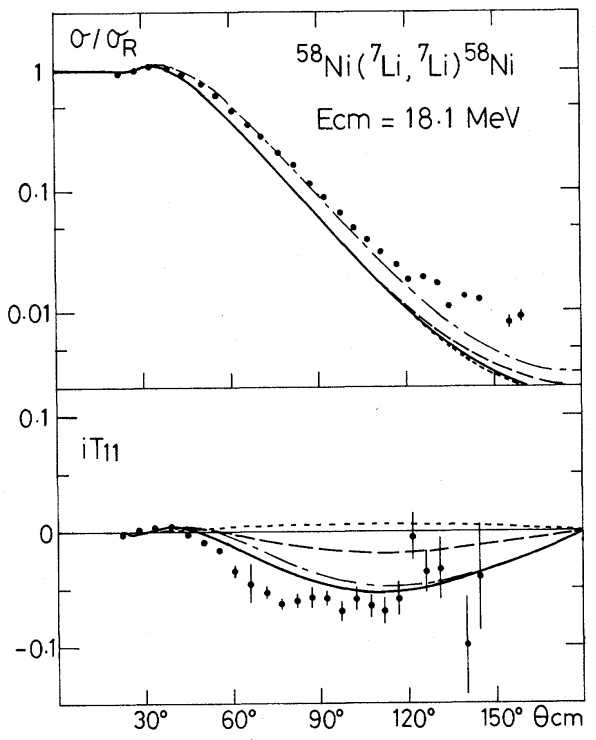

FIG. 2. Calculated results for ${ }^{7} \mathrm{Li}+{ }^{58} \mathrm{Ni}$ elastic scattering. The data are from Ref. 12. Dotted curves and dashed curves are the results of the one-channel calculation neglecting and including the rank-2 tensor potential, respectively. Solid curves and dash-dotted curves are the results of the coupled-channels and renormalized coupled-channels calculations, respectively. lated $i T_{11}$ is very small and has opposite sign to the experimental data. The dashed curves show the calculated results when the $T_{R}$ term is included. The sign of $i T_{11}$ is now negative but with a magnitude small compared to the data. The experimental tensor analyzing power data (not shown here) are reproduced very well.

Mukhopadhyay et al. concluded, ${ }^{20}$ in their folding-model calculation, that the rank-3 tensor term of the ${ }^{7} \mathrm{Li}-{ }^{58} \mathrm{Ni}$ interaction was important for obtaining the negative sign of $i T_{11}$. In contradiction, the present calculation shows that the rank-3 tensor term has a negligible effect not only on $i T_{11}$ but also on the other observables.

The results of the coupled-channels calculation for ${ }^{7} \mathrm{Li}+{ }^{58} \mathrm{Ni}$ are shown by the solid curves in Fig. 2. The inclusion of projectile excitation has a negative effect on $i T_{11}$ and agreement with the experimental data is considerably improved. The calculated inelastic cross section and $i T_{11}$ are also in good agreement with the data. Details of these and other coupled-channels calculations will be presented in a subsequent publication. ${ }^{15}$

Although obtaining a best fit to the elastic scattering data was not the primary object of the present work, the fact that the agreement of calculated and experimental cross sections is not quantitatively good might cause concern about the validity of the physical picture presented here. To clarify this, coupled-channels calculations were performed with the folding-model potentials renormalized so as to reproduce the cross-section data. The renormalization factor was 0.7 for the diagonal and $\mathbf{1 . 0}$ for the coupling nuclear potentials for ${ }^{6} \mathrm{Li}$ scattering, and 0.5 for the diagonal and coupling nuclear potentials in the ${ }^{7} \mathrm{Li}$ case. The Coulomb potentials were not renormalized. The results are shown by the dashdotted curves of Figs. 1 and 2. As shown there, the quality of fit to the experimental $i T_{11}$ is not altered. Other calculated elastic and inelastic observables for ${ }^{7} \mathrm{Li}+{ }^{58} \mathrm{Ni}$ scattering are not strongly affected by the potential renormalization.

Projectile excited states other than the first excited states were neglected in the present calculation. For ${ }^{6} \mathrm{Li}$ and ${ }^{7} \mathrm{Li}$ these have either different isospins or much higher excitation energies than the first excited states. At the incident energies under consideration, which are near the Coulomb barrier, it is appropriate to neglect them. Target excitation effects were implicitly included through the imaginary parts of the elementary cluster-target interactions. For a spin- 
less target, the target excitation mechanism alone does not generate an effective SO interaction for elastic scattering.

In summary, through parameter-free calculations it has been shown that the effect of projectile excitation produces large effects on elastic vector analyzing powers. These effects are of opposite sign in the cases of ${ }^{6} \mathrm{Li}$ and ${ }^{7} \mathrm{Li}$ scattering from ${ }^{58} \mathrm{Ni}$. This sign difference can be understood in the classical discussion presented. Also in ${ }^{7} \mathrm{Li}$ scattering the rank-2 tensor $\left(T_{R}\right)$ term of the interaction in the ground-state channel is found to be in part responsible for the agreement with the experimental $i T_{11}$. The folding-model SO potentials are found to play only a very minor role even for $i T_{11}$.

The classical discussion of the effect of projectile excitation can also be applied to other projectiles which have a well-developed cluster structure. For example, it predicts that projectile excitation will increase the strength of the SO interaction for ${ }^{19} \mathrm{~F}\left({ }^{16} \mathrm{O}+t\right)$ and ${ }^{13} \mathrm{C}\left({ }^{12} \mathrm{C}+n\right)$ projectiles without changing the sign from that of the folded SO potential. This is consistent with the findings of phenomenological analyses.9.11 It also predicts that projectile excitation will generate an effective SO potential of opposite sign to that predicted by the folded one for ${ }^{17} \mathrm{O}$ $\left({ }^{16} \mathrm{O}+n\right)$ and ${ }^{41} \mathrm{Ca}\left({ }^{40} \mathrm{Ca}+n\right)$ projectiles.

The calculations were performed with use of the optical-model code $\operatorname{LINA}^{21}$ and the coupledchannels code CHUCK3.$^{22}$ We would like to thank Dr. K. -H. Möbius and Dr. N. M. Clarke for providing us with copies of these programs. This work was supported by the Science and Engineering Research Council (U.K.).

(a) Present address.
${ }^{1}$ W. J. Thompson, in Proceedings of the Rochester Symposium on Heavy-Ion Elastic Scattering, Rochester, 1977, edited by R. M. DeVries (unpublished), p. 321, and references therein.

${ }^{2}$ H. Amakawa and K.-I. Kubo, Nucl. Phys. A266, 521 (1976).

${ }^{3} \mathrm{~W}$. Weiss et al, , Phys. Lett. 61B, 237 (1976).

${ }^{4}$ P. J. Moffa, Phys. Rev. C 16, 1431 (1977).

${ }^{5}$ F. Petrovich et al.; Phys. Rev. C 17, 1642 (1978).

${ }^{6}$ M. B. Golin and S. Kubono, Phys. Rev. C 20,1347 (1979).

${ }^{7}$ H. Nishioka, R. C. Johnson, and K.-I. Kubo, in Topics in Heavy Ion Reactions, compiled by A. K. Dhar, J. S. Lilley, and N. A. Nagarajan, Daresbury Laboratory, Warrington, Report No. DL/NUC/R21, 1981 (unpublished), p. 95 .

${ }^{8}$ P. Egelhof et al., Phys. Lett. 84B, 176 (1979).

${ }^{9}$ W. Dünnweber et al., Phys. Rev. Lett. 43,1642 (1979); B. F. Bayman, A. Dudek-Ellis, and P. J. Ellis, Nucl. Phys. A301, 141 (1979); Q. K. K. Liu and P. J. Ellis, Phys. Rev. C 222, 540 (1980); W. von Oertzen, private communication.

${ }^{10} \mathrm{P}$. Wust et al., Z. Phys. A 291, 151 (1979); W. von Oertzen et al., Z. Phys. A 301, 365 (1981).

${ }^{11}$ S. Kubono et al., Phys. Rev. Lett. 38, 817 (1977).

${ }^{12} \mathrm{G}$. Tungate et al., Phys. Lett. 98B, 347 (1981).

${ }^{13} \mathrm{H}$. Hasegawa and S. Nagata, Prog. Theor. Phys. 38 , 1188 (1967); R. E. Brown and Y. C. Tang, Phys. Rev. 176,1235 (1968).

${ }^{14}$ W. G. Love, T. Tersawa, and G. R. Satchler, Nucl. Phys. A291, 183 (1977).

${ }^{15}$ H. Nishioka, R. C. Johnson, J. A. Tostevin, and K.-I. Kubo, to be published.

${ }^{16}$ I. J. R. Griffith et al., Nucl. Phys. A146, 193 (1970).

${ }^{17}$ R. A. Hardekopf et al., Phys. Rev. C 21, 906 (1980).

${ }^{18}$ R. M. Del Vecchio and W. W. Daehnick, Phys. Rev. C 6, 2095 (1972).

${ }^{19}$ K.-I. Kubo and M. Hirata, Nucl. Phys. A187, 186 (1972).

${ }^{20}$ D. Mukhopadhyay, G. Grawert, D. Fick, and Z. Moroz, Phys.Lett. 104B, 361 (1981).

${ }^{21} \mathrm{G}$. Tungate and $\mathrm{Z}$. Moroz, private communication.

${ }^{22} \mathrm{~J}$. R. Comfort, private communication. 